\title{
MODELING THE REMARKABLE MULTIWAVELENGTH LIGHT CURVES OF EF ERIDANUS: THE DETECTION OF ITS IRRADIATED BROWN DWARF-LIKE SECONDARY STAR
}

\author{
THOMAS E. HARRISON ${ }^{1}$ \\ Department of Astronomy, New Mexico State University, Box 30001, MSC 4500, Las Cruces, NM 88003-8001; tharriso@nmsu.edu \\ STeve B. Howell ${ }^{2,3}$ and Mark E. Huber ${ }^{2}$ \\ Astrophysics Group, Planetary Science Institute, 620 North Sixth Avenue, Tucson, AZ 85705; howell@psi.edu, mhuber@psi.edu \\ Heather L. Osborne ${ }^{1}$ and Jon A. Holtzman \\ Department of Astronomy, New Mexico State University, Box 30001, MSC 4500, Las Cruces, NM 88003-8001; \\ hosborne@nmsu.edu,holtz@nmsu.edu \\ JENNIFER L. CASH \\ Department of Physical Sciences, South Carolina State University, P.O. Box 7024, Orangeburg, SC 29117; jcash@physics.scsu.edu \\ AND \\ DaWn M. GeLino \\ Center for Astrophysics and Space Sciences, University of California, San Diego, 9500 Gilman Drive, La Jolla, CA 92093-0424; \\ dgelino@ucsd.edu \\ Received 2002 July 1; accepted 2002 February 12
}

\begin{abstract}
We present optical and infrared phase-resolved photometry of the magnetic cataclysmic variable EF Eri during a low state. The $B V R I J$ light curves are very similar in appearance; all exhibit a dip of a few tenths of a magnitude near binary orbital phase 0.5 . In contrast, however, the $H$ - and $K$-band light curves show very large modulations, with maxima at orbital phase 0.5 . We show that these modulations are not due to ellipsoidal variations but to the reflection/heating of one face of the very cool secondary star by the white dwarf primary. We find that the dips in the BVRIJ light curves are best modeled by a single hot spot on the white dwarf primary that is self-eclipsed once per orbit. By using an orbital inclination of $i=45^{\circ}$, we find the colatitude of the hot spot is $35^{\circ} \pm 10^{\circ}$, its temperature is $12,000 \mathrm{~K}$, and its radius is $29^{\circ} \pm 15^{\circ}$. To explain the larger minimum seen in the $J$-band light curve requires that $\sim 20 \%$ of the observed flux be supplied by an additional source of luminosity, which we believe is residual cyclotron emission. To model the observed amplitudes in the $H$-and $K$-band light curves requires a very cool irradiated brown dwarf-like secondary star with temperature $T_{\text {eff }} \approx 900 \mathrm{~K}$ (for $i=45^{\circ}$ ). The irradiated side of this secondary star, however, has $T_{\text {eff }} \lesssim 1600 \mathrm{~K}$. We present a new $K$-band spectrum of EF Eri that is consistent with this result. To properly model the light curve of EF Eri will require the incorporation of brown dwarf atmospheres into the WilsonDivinney program, as well as better estimates for the irradiated limb-darkening coefficients and albedos of such objects. Finally, we present a three-dimensional hydrodynamic model for EF Eri that demonstrates that the accretion stream from the secondary star is almost fully controlled by the magnetic field of the white dwarf primary. This model predicts that the narrow dips observed in the high-state infrared light curves of EF Eri, which nominally should lead the binary in orbital phase, can occur very close to phase 0.0 , as required by our light-curve modeling.
\end{abstract}

Key words: binaries: close — stars: individual (EF Eridani) — stars: low-mass, brown dwarfs — stars: magnetic fields — stars: variables: general

\section{INTRODUCTION}

EF Eri is an ultrashort-period (81.0 minutes; Bailey et al. 1982) magnetic cataclysmic variable (CV) of the AM Herculis type (polar). Ferrario, Bailey, \& Wickramasinghe (1996) modeled the cyclotron humps seen in infrared spectroscopy to determine magnetic field strengths of 16.5 and $21 \mathrm{MG}$ for two separate cyclotron emission regions.

\footnotetext{
${ }^{1}$ Visiting Astronomer, Kitt Peak National Observatory, National Optical Astronomy Observatory, which is operated by the Association of Universities for Research in Astronomy, Inc., under cooperative agreement with the National Science Foundation.

${ }^{2}$ Visiting Astronomer, United Kingdom Infrared Telescope. The United Kingdom Infrared Telescope is operated by the Joint Astronomy Centre on behalf of the UK Particle Physics and Astronomy Research Council.

${ }^{3}$ Current address: Institute of Geophysics and Planetary Physics, 1432 Geology Building, University of California, Riverside, CA, 92521; steve.howell@ucr.edu.
}

Östreicher et al. (1990) arrived at a value of 10 MG for the field in EF Eri through the observation of the polarized Zeeman components of $\mathrm{H} \alpha$. When in its high state, EF Eri exhibits all the phenomena normally associated with AM Herculis variables, such as highly variable optical fluxes, orbitally modulated linear and circular polarization, strong hydrogen and helium line emission, and both long- and short-term X-ray variations. In 1997 December, EF Eri was found to be in its low state, having faded from $V=14$ to $V=18$ (Wheatley \& Ramsey 1998). Optical spectra at this time demonstrated that the photosphere of the white dwarf primary had been detected: broad $\mathrm{H}$ I Balmer absorption profiles were seen. Also seen were the absorption components arising from Zeeman splitting in the intense magnetic field of the white dwarf. Wheatley \& Ramsey estimated a magnetic field strength of $14 \mathrm{MG}$, consistent with the two previous estimates for the magnetic field strength in EF Eri. 
Beuermann et al. (2000) have used the Wheatley \& Ramsey spectrum to derive additional parameters for EF Eri. They found that the optical spectrum was consistent with that of a white dwarf at a temperature of $9500 \mathrm{~K}$. Orbital light modulations implied an additional component, which they suggest was due to a hot spot at a temperature of $15,000 \mathrm{~K}$ that covered $6 \%$ of the white dwarf surface.

Beuermann et al. found no evidence for the detection of a secondary star in the optical spectrum of EF Eri. Investigating a variety of scenarios (primary mass, radius, and distance), they suggest that the spectral type of the secondary in EF Eri is M9 or later. Howell \& Ciardi (2001) present an infrared spectrum of EF Eri in which they believe that they have detected the secondary and that it has an effective spectral type near L4, with $T_{\text {eff }} \sim 1650 \mathrm{~K}$. We have obtained a new $K$-band spectrum and find a clear detection of a very late-type secondary star $(\geq \mathrm{M} 8 \mathrm{~V})$. This very late spectral type is consistent with theoretical models for the secondary stars of ultrashort-period CVs that predict masses in the range 0.02 to $0.06 M_{\odot}$ and effective temperatures below 2000 K (Howell, Nelson, \& Rappaport 2001b).

Below we present new simultaneous phase-resolved multiwavelength optical and infrared photometry of EF Eri during a low state. We believe the interpretation of these new data further strengthen the case for the presence of a very cool secondary star ( $\left.T_{\text {eff }} \lesssim 1200 \mathrm{~K}\right)$. In the next section, we describe our observations, followed by a discussion of the current state of EF Eri in $\S 3$. We then model the light curves of EF Eri in $\S 4$, followed by a summary of our results in $\S 5$.

\section{OBSERVATIONS}

\subsection{Photometry}

Infrared photometry of EF Eri was obtained using the Simultaneous Quad Infrared Imaging Device $\left(\right.$ SQIID $\left.^{4}\right)$ on the KPNO $2.1 \mathrm{~m}$ telescope. SQIID allows for simultaneous observations in $J H K$ and in a narrowband $L$ filter (EF Eri was much too faint to have been detected in the narrow $L$-band filter). EF Eri was observed with SQIID on 2001 December 2 from 0400 UT to 0700 UT, covering two full orbital cycles. Because of the necessity of keeping all the exposure times the same when using SQIID, the maximum allowable exposure time is determined by the background in the $K$ band and the desire to operate in the linear regime of the detector. For EF Eri, the individual exposure times were $10 \mathrm{~s}$. An observation consisted of five co-added $10 \mathrm{~s}$ exposures (at position A), a beam switch to a position $26^{\prime \prime}$ west (position B), a pair of co-added exposures, and a beam switch back to the original position (an ABBA sequence). A single observational sequence took about 4 minutes to complete. Difference images of the average of the data at the A and $\mathrm{B}$ positions were constructed, and these images were then flat-fielded. Aperture photometry was performed using the normal IRAF procedures, and the differential magnitudes and UT times at the A and B positions were averaged to produce the $J H K$ light curves. Finally, these data were phased using the Bailey et al. (1982) ephemeris and put on the photometric system of Bessell \& Brett (1988) by using data from the 2MASS database for the four differential photometry reference stars, and the transformation equations from Carpenter (2001). The result is shown in Figure 1

\footnotetext{
${ }^{4}$ See http://www.noao.edu/kpno/sqiid/sqiidmanual.html.
}
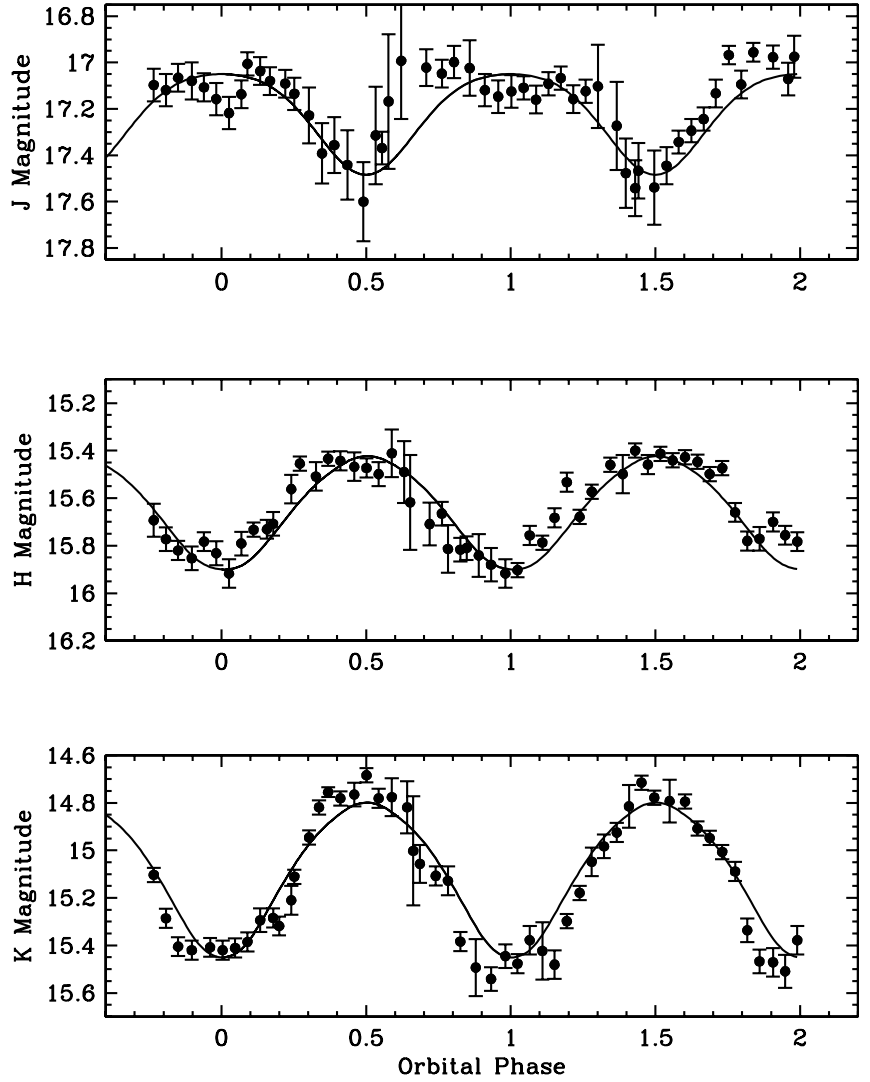

FIG. 1.-JHK light curves of EF Eri, showing our final model for EF Eri (solid lines). The light curves have been phased using the ephemeris derived by Bailey et al. (1982).

(the model fits to the light curves in this figure are discussed in $\S 4.5$ ). It was photometric during most of the $3 \mathrm{hr}$ sequence of SQIID observations, but there were two brief intervals of light cirrus. One of these occurred near binary orbital phase 0.6 , and the other at phase 1.3. These events are most clearly seen in the $J$-band light curve during which EF Eri was occasionally difficult (or impossible) to detect, and the error bars on our photometry are substantially larger during these times.

At nearly the same time (0341 to 0646 UT) as the infrared observations, EF Eri was observed in automated mode using the New Mexico State University $1.0 \mathrm{~m}$ telescope. The NMSU $1.0 \mathrm{~m}$ telescope is equipped with an apogee $512 \times 512$ thinned, back-side-illuminated CCD with standard Johnson-Cousins BVRI filters. The optical data set consists of 3 minute exposures in each filter, done sequentially in the order $B, V, R$, and $I$. These data were processed in the normal way, and a differential light curve for EF Eri was generated by comparison with four nearby stars. The light curves were put on the standard system by using Landolt standards to calibrate the differential photometry reference stars. The light curves shown in Figure 2 have been phased using the Bailey et al. (1982) ephemeris (the models plotted in these figures are discussed in $\S 4.4$ ). The signal-tonoise ratios $(\mathrm{S} / \mathrm{Ns})$ of these data were affected by the presence of a nearly full Moon located $\approx 70^{\circ}$ from EF Eri.

\subsection{Infrared and Optical Spectroscopy}

Time-resolved $K$-band spectroscopy of EF Eri was performed during 2000 December and 2001 October at the 


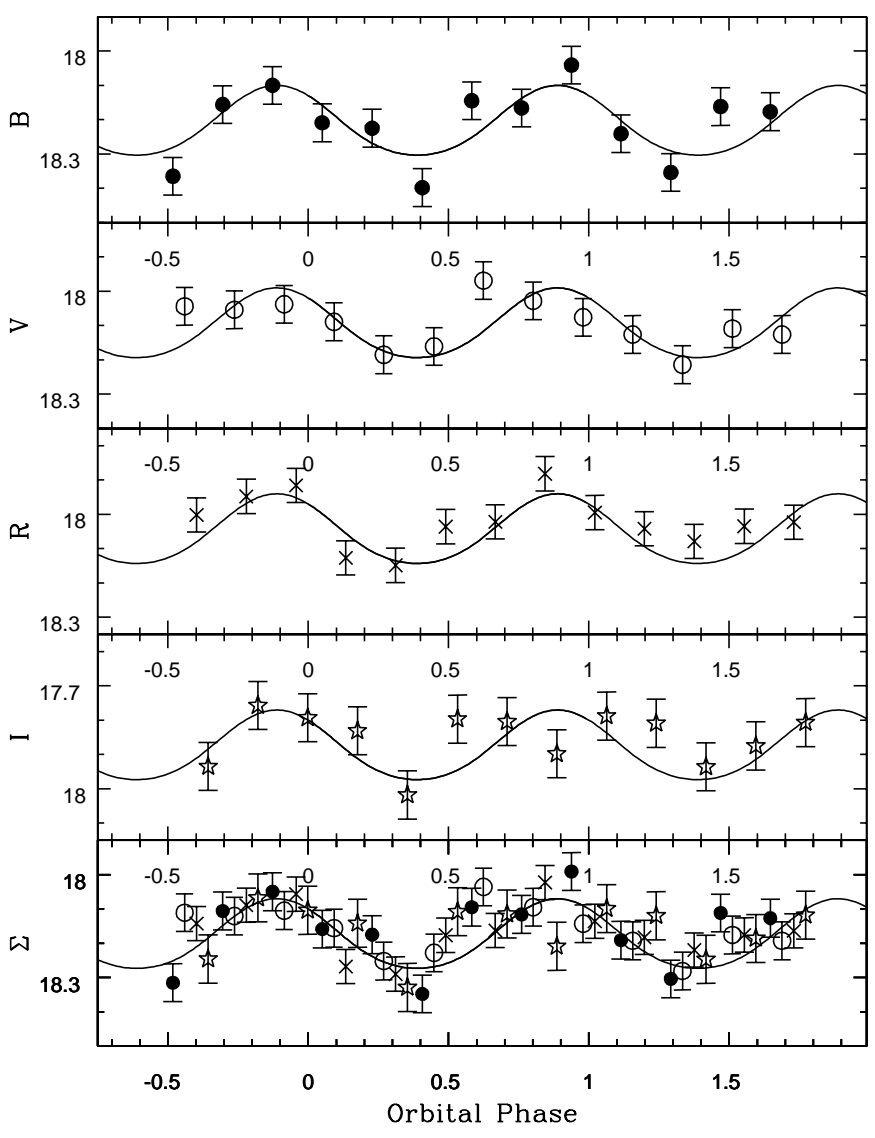

FIG. 2.-Top, second, third, and fourth: Optical (BVRI) light curves of EF Eri. The phasing is the same as in Fig. 1. Bottom: Sum of the four light curves, produced by giving each of the individual light curves a small zeropoint offset. This is possible because the optical colors of EF Eri are similar $(B-V \approx V-R \approx V-I \approx 0)$ and do not change significantly over an orbital cycle. The solid line in all panels is our final model for EF Eri. The minima in these light curves for the best-fitting model occur at an orbital phase of 0.39 .

United Kingdom IR Telescope (UKIRT), located on Mauna Kea. Initial analysis of the 2000 December spectra were presented in Howell \& Ciardi (2001). The 2001 October spectra were obtained using the cooled grating spectrograph (CGS4) and covered the wavelength range from 1.85 to $2.47 \mu \mathrm{m}$. The spectral region shortward of $\sim 1.9$ $\mu \mathrm{m}$ was unusable because of strong telluric absorption. We used the 40 line $\mathrm{mm}^{-1}$ grating and a slit width of 2 pixels (1".2), giving a resolution of $24 \AA$ pixel $^{-1}\left(325 \mathrm{~km} \mathrm{~s}^{-1}\right)$. These spectra were reduced in the normal manner, that is, biasand flat-field-corrected and wavelength-calibrated using an argon arc lamp, removal of bad pixels, and linearization of the data. The correction for telluric absorption was accomplished using observations of A-type stars near in time and position (after patching hydrogen lines out of their spectra). Each individual spectrum was then optimally extracted (including removal of any optical curvature) and fluxcalibrated using observations of UKIRT standard stars obtained before and after each series. The resulting spectrum, a median of the 19 individual spectra smoothed by 5 pixels, is shown in Figure 3, where it is compared with that of an M8 V template. Because of the orbital motion of EF Eri and because the medianed data set covers one complete orbital period, the spectrum shown in Figure 3 suffers from orbital smearing. For example, by using $M_{1}=0.6 M_{\odot}$, a mass ratio of $q \equiv M_{2} / M_{1}=0.1$, an orbital inclination of $45^{\circ}$, and an orbital period of 81 minutes, we estimate a radial velocity semiamplitude of $K_{2}=340 \mathrm{~km} \mathrm{~s}^{-1}$. This motion, however, is effectively unresolvable given the relatively low dispersion of the UKIRT spectrum.

On 2001 March 11 we observed EF Eri by using the Cerro Tololo Inter-American Observatory (CTIO) $1.5 \mathrm{~m}$ with the Cassegrain spectrograph. The instrumental setup included the 300 line $\mathrm{mm}^{-1}$ grating (No. 9), delivering a resolution of $2.87 \AA$ pixel $^{-1}$. Four 20 minute integrations were combined to produce the spectrum shown in Figure 4. Since these data cover one complete orbital period, the optical spectrum of EF Eri should also suffer from orbital smearing, but given that we believe the optical spectrum to be that of the white dwarf primary, this effect should be significantly smaller $\left(K_{1} \approx 34 \mathrm{~km} \mathrm{~s}^{-1}\right)$ because of the expected mass ratio of EF Eri. This motion would be completely unresolved with the spectral resolution employed in our observations.

\section{COMPARISON OF EF ERI IN ITS HIGH AND LOW STATES}

With an average $V$ magnitude of 18.1, the optical photometry clearly demonstrates that EF Eri was still firmly in its low state at the end of 2001. The dramatic weakening of the accretion activity between high and low states can be seen by comparing the new low state $K$-band spectrum of EF Eri with the high-state spectrum obtained by Ferrario et al. (1996, their Fig. 1). In the high-state $K$-band spectrum, there is a large cyclotron hump at $2.15 \mu \mathrm{m}$, as well as strong $\mathrm{H} \mathrm{I}(\mathrm{Br} \gamma)$ emission. These features were not present in either the 2000 December (Howell \& Ciardi 2001) spectrum or in our 2001 October data. In Figure 3, we have compared the new $K$-band spectrum of EF Eri with that of the M8 V $\left(T_{\text {eff }} \approx 2200 \mathrm{~K}\right.$ ) dwarf VB 10 (from Harrison et al. 2003). Given the low $\mathrm{S} / \mathrm{N}$ of the EF Eri spectrum, there are only two obvious features: the decrement in flux longward of $2.29 \mu \mathrm{m}$ (due to a combination of $\mathrm{CO}$ and $\mathrm{H}_{2} \mathrm{O}$ absorption) and the slight blueward rise below $2.08 \mu \mathrm{m}$. As can be seen in the comparison with VB 10, the continuua of the two objects match very well between 2.20 and $2.40 \mu \mathrm{m}$, indicating that we have truly detected the late-type secondary star in EF Eri. There are several other weak features in the spectrum of EF Eri that may correspond to atomic lines, but none are sufficiently strong for us to confidently claim detections.

The optical spectrum presented in Figure 4 suggests that EF Eri has continued to evolve to an even lower activity level than that found by Wheatley \& Ramsey (1998). The 2001 March spectrum reveals a dramatically weakened hydrogen $\mathrm{H} \beta$ emission line compared with the spectrum of EF Eri obtained four years earlier (Fig. 1 of Wheatley \& Ramsey 1998): the peak of the emission component of $\mathrm{H} \beta$ now lies below the local continuum. For comparison, we have overlaid (without scaling) the $9500 \mathrm{~K}$ nonmagnetic white dwarf model spectrum used by Beuermann et al. (2000, kindly provided by B. Gänsicke) to model the Wheatley \& Ramsey spectrum of EF Eri. This shows that there has not been a significant change in the continuum level in the intervening four years.

The change in the spectral energy distribution (SED) of EF Eri between high and low states has been dramatic. In Figure 5 we present the high-state SED observed by Bailey et al. (1982) in 1979 December. In this figure we also include 


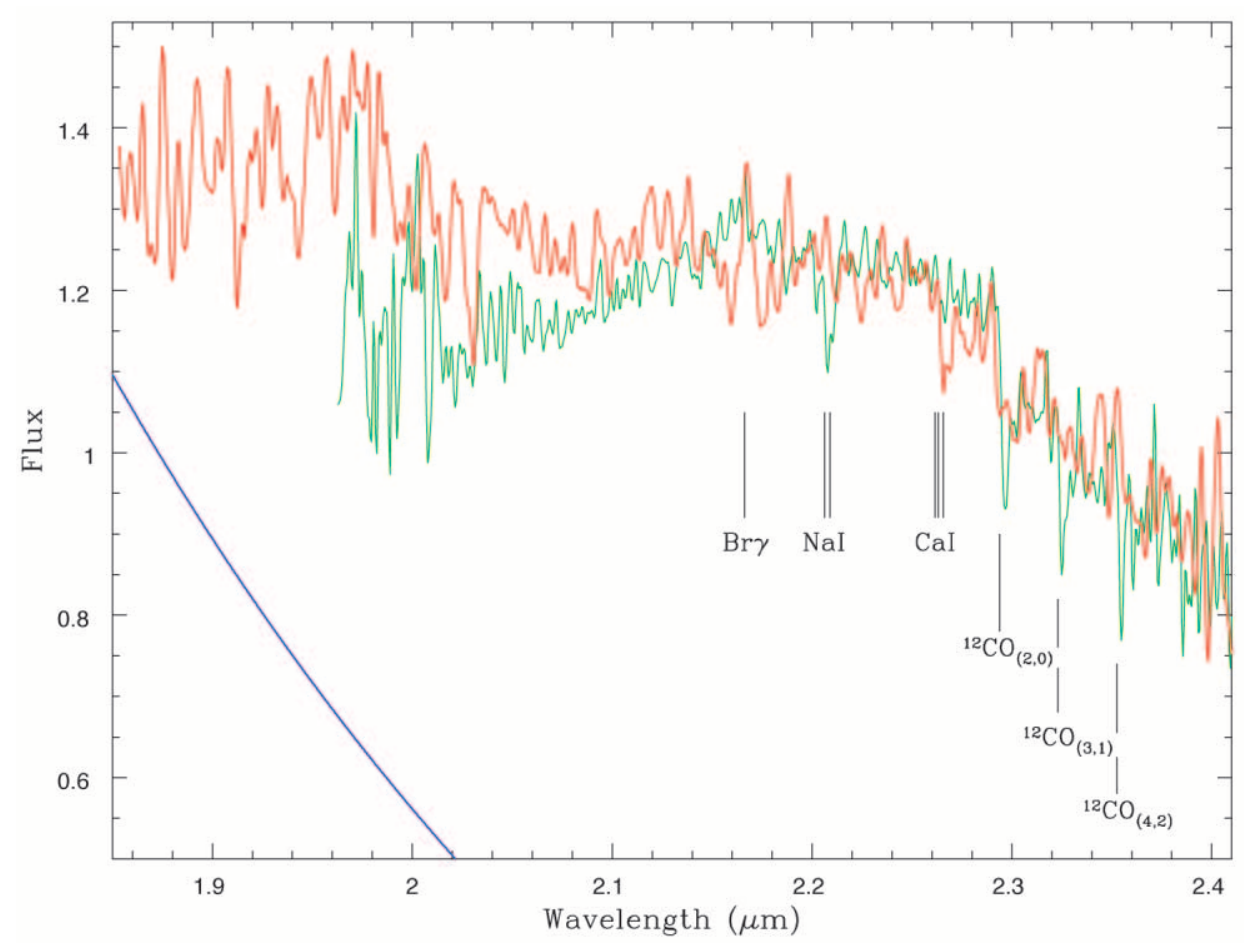

FIG. 3.- Infrared spectrum of EF Eri obtained at UKIRT in 2001 October (red), compared with that of the M8 dwarf VB 10 (green; obtained with SPEX on the IRTF, from Harrison et al. 2003). The continuua match very well between 2.2 and $2.4 \mu \mathrm{m}$. The sharp decline beyond $2.29 \mu \mathrm{m}$ is due to overlapping CO absorption features (the bandhead wavelengths of the most prominent of these have been identified), in combination with water vapor absorption. Unlike the spectra of late-type stars (e.g., VB 10), the spectrum of EF Eri has a gentle rise below $2.08 \mu \mathrm{m}$. The blue line in this figure represents a $9500 \mathrm{~K}$ blackbody that supplies $7.5 \%$ of the $K$-band flux (the orbitally averaged contribution from the white dwarf primary). The sum of a late-type stellar spectrum and the flux from the white dwarf primary can explain the blueward rise seen in the $K$-band spectrum of EF Eri.

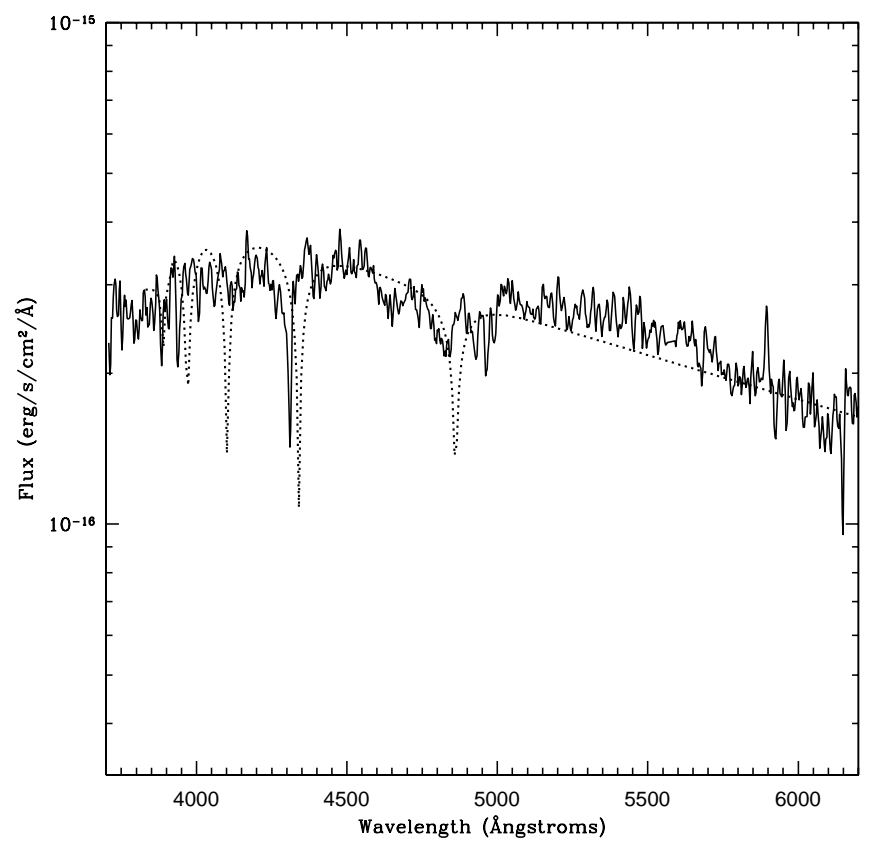

FIG. 4.-Optical spectrum of EF Eri obtained using the CTIO $1.5 \mathrm{~m}$ telescope on 2001 March 11, showing the white dwarf model used by Beuermann et al. (2000) for EF Eri (dotted line). The spectrum is the sum of four individual 20 minute exposures. The overall continuum has not changed significantly in the four years since the spectrum obtained by Wheatley \& Ramsey (1998). The $\mathrm{H} \beta$ emission line, however, has weakened substantially, with its peak now falling below the local continuum.

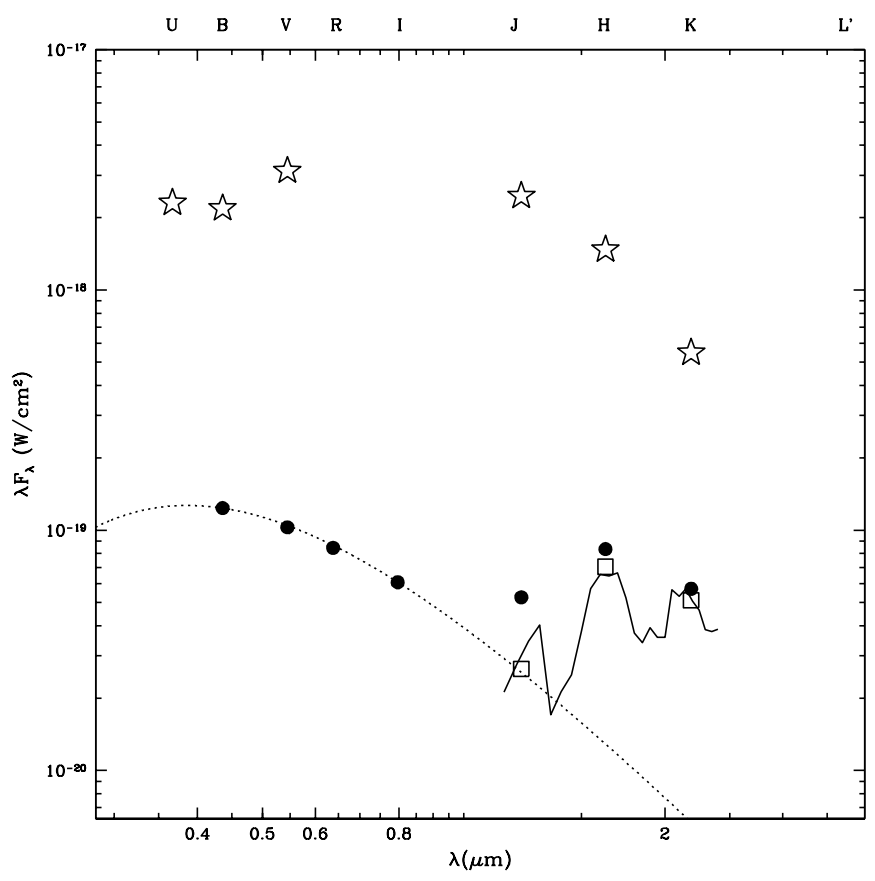

FIG. 5.-High-state (stars) and low-state (circles) spectral energy distributions (SEDs) of EF Eri. EF Eri is a factor $\sim 30$ fainter in its low state. The low-state SED is composed of the optical fluxes at their minima (to exclude the contribution of the photospheric hot spot). The infrared data are from phase 0.0 (the nonirradiated hemisphere of the companion). The $B V R I$ data are extremely well modeled by using a $9500 \mathrm{~K}$ blackbody (dotted line). We have subtracted the $\mathrm{J}_{-}, \mathrm{H}$-, and $\mathrm{K}$-band contributions of this blackbody from the infrared photometry to remove the contribution of the white dwarf. The residual SED (squares) is very red and is similar in shape to that of an L8 V (solid line; adapted from McLean et al. 2001). 
a minimum-light SED using our new data. The overall SED has declined by a factor of $\sim 30$ between the high and low states. As we will describe in the next section, we believe the dips in the $B V R I$ light curves are due to a hot spot on the white dwarf photosphere that is self-eclipsed once per orbit; thus, to best model the temperature of the underlying white dwarf, we use the fluxes when this spot contaminates the observed SED (orbital phase $\sim 0.4$ ) very little or not at all. The resulting $B V R I$ data are perfectly modeled by a $9500 \mathrm{~K}$ blackbody. To highlight the contribution of the secondary star to this SED, the $J-, H$-, and $K$-band fluxes in this plot are those at phase 0.0 (the nonirradiated hemisphere of the companion, as described below). To further examine the nature of the secondary star, we subtract the white dwarf contribution (dotted line) from the observed JHK photometry to produce the open squares plotted in Figure 5. The white dwarf-subtracted infrared colors at phase 0.0 are $J-K=1.70$ and $H-K=0.42$, consistent with those of a late-type $\mathrm{L}$ dwarf, as is demonstrated by the comparison of the observed SED with the spectrum of an L8 V. It is important to note that because of the presence of weak cyclotron emission in this system, the infrared colors are contaminated, and the secondary star may be redder than the phase 0.0 values just derived.

The optical and infrared light curves of EF Eri are dramatically different between high and low states. Bailey et al. used a sharp dip (having a total duration of $\approx 5$ minutes) seen in their infrared light curves, most clearly in the $J$ band, to define the zero point of the phasing. This dip also coincided with a similar minimum in the X-ray light curve. The white-light temporally resolved circular polarimetry of Bailey et al. had a maximum in polarization at this zerophase point. They found that a model in which the accretion column points directly toward us at phase 0 and away from us at phase 0.5 best explains their data. In this scenario the sharp dip in the infrared light curves is caused by an eclipse of the accretion region by the narrow accretion stream, a common feature of polars (see Sirk \& Howell 1998); thus in this scenario the phasing of the light curves is related to the location of the accretion stream and accretion region and is not strictly related to the binary orbital phase. It is interesting to note that we also see evidence for a short-lived minimum in our $J$-band light curve at phase 0.0. This feature, however, was not clearly present one orbit later. Bailey et al. found that the narrow dips did not reliably recur every orbit. Because of the long effective integration times of the individual data points plotted in Figure 1, short-lived features, such as the narrow high-state dips, would not be properly resolved.

The high-state $J$-band light curves of EF Eri observed by Bailey et al. had considerable structure, with a variety of transient features. One feature that did persist, however, was a deep $\left(\Delta m_{J} \approx 1 \mathrm{mag}\right)$ broad dip centered near phase 0.4 . Bailey et al. suggested that this feature was the selfeclipse of the accretion region on or near the white dwarf surface, though in their phasing scenario they concluded that it required some asymmetry in the magnetic field geometry to prevent it from occurring at phase 0.5 . Our $J$-band light curve (see Fig. 1) reveals a narrower and shallower dip centered near phase 0.5 . The profile of this dip does not appear symmetric, but the egress of the first eclipse and the ingress of the second were hampered by thin cirrus (as indicated by the larger error bars on the data at phases 0.6 and 1.3). Given the likelihood that the properties of the accre- tion region could change between the high and low states, it appears reasonable to associate the $J$-band dip in the low state with the feature seen in the high state, even though they appear to have slightly different phases. The high- and lowstate light curves in the $H$ and $K$ bands do not have any features in common.

The optical light curves of EF Eri in its high state presented by Bailey et al. (1982) are quite complex, with shorttimescale variability of $\pm 0.2 \mathrm{mag}$. The high-state $U B V$ light curves displayed a very broad minimum centered near phase 0.0 . Our new low-state $B V R I$ light curves are relatively smooth, with little evidence for transient behavior. There is a single broad dip of $\approx 0.2$ mag once per orbit seen in all four filters. As shown in Figure 2 and to be discussed in the next section, we were able to successfully model this feature with a single hot spot on the white dwarf. The best-fitting model puts the minima of the optical dips at phase 0.39 , similar to the location of the broad dip seen in the high-state $J$-band light curves obtained by Bailey et al.

\section{MODELING THE LIGHT CURVES OF EF ERI}

We have used the most recent version of the Wilson-Divinney light-curve program (Wilson 1998, hereafter WD98) to model our multiwavelength data set for EF Eri. A description of the application of this program to model the magnetic system AR UMa can be found in Howell, Gelino, \& Harrison (2001a), and models for the ellipsoidal variations in the soft X-ray transient systems GU Mus and V616 Mon generated using WD98 can be found in Gelino, Harrison, \& McNamara (2001a) and Gelino, Harrison, \& Orosz (2001b), respectively. As discussed in those papers, a variety of intrinsic system parameters control the light curves of interacting binaries. Among the most important of these are the orbital inclination angle and the relative temperatures and luminosities of the two components. With sufficient data, it is possible to deconvolve just about all the relevant parameters for the binary star system from multiwavelength light curves. In reality, however, the process is greatly accelerated if some input parameters, such as the primary star mass and temperature, are known ahead of time.

To explain the unusual light curves of EF Eri required us to apply WD98 in a regime in which a number of the model parameters for the very cool secondary star are not known. For example, WD98 incorporates Kurucz atmospheres to create realistic models for binary star light curves. Unfortunately, atmosphere models with effective temperatures below $3500 \mathrm{~K}$ are not presently included in WD98. Additional important parameters for light curve-modeling, such as a specification of the albedo, the gravity-darkening relationship, and limb-darkening coefficients, do not currently exist for stars with temperatures below $2000 \mathrm{~K}$. As we will show, even if they did exist we would need to know how the effect of irradiation by the primary star changes these parameters; thus, our limited knowledge of the values for required input parameters forces us to constrain beforehand as many system parameters as possible.

\subsection{White Dwarf Primary}

As found by Beuermann et al. (2000) and confirmed by the SED presented in Figure 5, the white dwarf in EF Eri is well modeled by a blackbody with an effective temperature of $9500 \mathrm{~K}$. Wheatley \& Ramsay (1998) noted that the 
optical light curve of EF Eri showed modulations of \pm 0.2 mag. Presumably, those variations were of the same sort that we have detected. Beuermann et al. (2000) suggest that such modulations could be adequately explained by a hot spot (presumably the accretion region) with $T_{\text {eff }}=15,000 \mathrm{~K}$ that covered $6 \%$ of the white dwarf surface. We have incorporated this suggestion into our modeling efforts, allowing both the areal coverage and effective temperature of this spot to be adjusted until a best fit of the optical data was achieved. The latitude and longitude of this spot were also allowed to vary to achieve the best fit. The mass and radius of the white dwarf primary enter into the light-curve modeling program through the gravitational potential. The white dwarf mass was set to $0.6 M_{\odot}$ (Howell et al. 2002) such that the primary had an effective gravity of $\log g=8.0$, giving a radius of $R_{\mathrm{WD}}=8.2 \times 10^{8} \mathrm{~cm}$. We kept these latter two quantities and the effective temperature of the white dwarf fixed throughout the modeling process. It is important to note that we have also assumed that the white dwarf is phase-locked in corotation with the binary as is the usual case in polars.

\subsection{Secondary Star}

Beuermann et al. (2000), Howell \& Ciardi (2001), and Harrison, Johnson, \& Howell (2002) provide compelling evidence that the secondary star of EF Eri is a brown dwarf-like object having a mass of $\lesssim 0.06 M_{\odot}$ and an effective temperature of $T_{\text {eff }} \lesssim 1600 \mathrm{~K}$. Our new $K$-band spectrum of EF Eri is consistent with this conclusion. However, as is demonstrated in Figure 6 and found in our final models described below, the infrared spectrum presented in Figure 3 essentially represents only that of the irradiated hemisphere of the secondary star in EF Eri and not the true spectrum of the underlying object (being heavily weighted in $\mathrm{S} / \mathrm{N}$ toward the times when EF Eri is at its brightest, phase 0.5). Obviously, we will have to allow the temperature of the secondary star to vary during the modeling process.

Without a determination of the spectral type of the secondary star, it is difficult to estimate its mass $\left(M_{2}\right)$. For light-curve modeling, it is important to have a reasonable estimate of the mass ratio $\left(q \equiv M_{2} / M_{1}\right)$ of the two stellar components, so that we can define the geometry of the binary system, though, as discussed by Gelino et al. (2001a), the assumed mass ratio of the binary has relatively minor effects on the shape and amplitudes of the resulting light curves. Based on the evolutionary considerations from Howell et al. (2001b), we have decided to assume that the secondary star has a mass near $0.06 M_{\odot}$, resulting in $q=0.10$. We also assume that the secondary star continues to completely fill its Roche lobe, even though the mass transfer rate appears to be very low or nonexistent at the time of our observations. The direct cause of the low states in polars and other CVs is still being debated, but it appears that a change in the radii of the secondary stars is probably not a viable explanation (King \& Cannizzo 1998, but see Howell et al. 2000).

\subsection{Orbital Period and Inclination}

Bailey et al. (1982) used new and existing observations to derive an 81 minute orbital period and an ephemeris for EF Eri by using the sharp $J$-band and X-ray minima. Additional evidence that Bailey et al. have correctly identified the true orbital period is that the circular polarization repeats on an 81 minute period; thus, the interpretation of the low- state $H$ - and $K$-band light curves seen in Figure 1 as due to ellipsoidal variations arising from the motion of the distorted secondary star (which would have two maxima and minima per orbital period) can be confidently rejected.

Light-curve modeling in noneclipsing binaries, which have ellipsoidal variations from the secondary star, is the best available method for determining the orbital inclination angle. Since we have rejected this interpretation for the observed modulations in EF Eri, it is more problematic to allow the orbital inclination to be a completely free parameter in the modeling process; thus, we thought a more robust procedure would be to enter the modeling process with a constrained range of possible orbital inclinations. Bailey et al. (1982) settle on a binary orbital inclination of 45.8 and cite several earlier sources that estimate the orbital inclination of EF Eri to be near $45^{\circ}$. Cropper (1985) found an inclination of $70^{\circ} \pm 10^{\circ}$, whereas Cropper (1990) weighted these values equally, giving $i=58 \pm 12^{\circ}$. These values for the orbital inclination were useful starting points in the modeling process.

\subsection{Modeling the Optical Light Curves with WD98}

As noted earlier, there is very little evidence for contamination of the optical data by the secondary star. If we were to fit a two-blackbody model, a $9500 \mathrm{~K}$ white dwarf plus a $2000 \mathrm{~K}$ secondary, to the minimum light SED shown in Figure 5, we would find that the contamination in the $I$ band remains below 10\%. Our final model for EF Eri will actually have a much cooler secondary star than this two-blackbody model, and therefore the $I$-band contamination proves to be negligible; thus, the physical parameters of the secondary star do not have to be accurately known to model the optical light curves. What we need to specify is the temperature of the primary, the orbital inclination angle, and the temperature, area, longitude, and latitude of the photospheric/accretion region hot spot (hereafter, simply the hot spot). We have set the bolometric albedo of the white dwarf to 1.0 and used limb-darkening coefficients appropriate for a $9500 \mathrm{~K}$ atmosphere but calculated for $\log g=5.0$ (the largest gravity atmosphere incorporated into WD98). While these are not the exact parameters appropriate for the white dwarf atmosphere used in our light-curve modeling of EF Eri, use of the exact values is relatively unimportant given the spherical nature of the primary.

It is obvious that a variety of combinations of hot spot temperature, areal coverage, and spot locations can be contrived to give similar results. With the limited $\mathrm{S} / \mathrm{N}$ of our observations, we cannot tightly constrain the nature of the hot spot. The amplitude of the optical variations is mostly fixed by the temperature and areal coverage of the hot spot, less so by its apparent latitude (here we mean the sum of the orbital inclination and the magnetic pole colatitude). The phase at which the dips occur, however, is sensitive only to the longitude of the hot spot. We first constructed a model with a spot that could reproduce the amplitude of the variations and then varied the longitude of the spot. Since the exact phase of the optical dips is not clear from any of the single-bandpass light curves, we offset and co-added all the optical data together (Fig. 2, bottom) to increase our phase coverage to better locate the dip centers. The model with the lowest (reduced) $\chi_{\nu}^{2}$ value has the dips centered at phase 0.39 , corresponding to a spot on the white dwarf with an 
"orbital phase" of 0.89 . This longitude was not affected by changes in either the colatitude of the hot spot or the binary orbital inclination.

With the longitude of the spot fixed, we then attempted to constrain the colatitude $(\beta)$, orbital inclination $(i)$, areal coverage, and temperature of the spot. The colatitude and orbital inclination have their greatest effect on the shape of the minima, as they determine how long the spot is in or out of view. If the spot is out of view for a long time, the model light-curve minima have flat bottoms. Even with the modest $\mathrm{S} / \mathrm{N}$ of the light curves seen in Figure 2, it is apparent that the minima do not have very flat bottoms. Note that the size of the spot also affects the duration of the self-eclipse. Spots with large areal coverage can remain in view throughout a rotational/orbital cycle given certain values for the orbital inclination and colatitude. For this test, we constrained the spot radius so that the total coverage was $6 \%$ of the white dwarf's surface. We then varied both the orbital inclination and magnetic colatitude over the range $30^{\circ}$ to $58^{\circ}$. It was quickly apparent that models in which the sum of these two angles was greater than $80^{\circ}$ produced light curves with flat minima. Our final best-fitting model has $i=35^{\circ}$ and $\beta=35^{\circ}$. Changing either of these by $10^{\circ}$ increased the $\chi_{\nu}^{2}$, but given the $\mathrm{S} / \mathrm{N}$ of the data such models cannot be excluded (especially given the influence of the spot size on the light-curve shape). Models with $i \gtrsim 45^{\circ}$ and $\beta \gtrsim 45^{\circ}$, however, produce light curves with very flat minima. Given the earlier results on the binary inclination of the system, we will adopt the values $i=45^{\circ}$ and $\beta=35^{\circ}$ in what follows.

Constraining the hot spot radius and temperature is more difficult. In fact, it is likely that the accretion region cannot be specified with a single temperature, since it must eventually blend in with the surrounding photosphere at its edges. Given this caveat, however, it is easy to reproduce the lightcurve amplitude with a small single-temperature hot spot or a larger slightly cooler spot. To attempt to derive these two parameters, we ran models over the range $11.5<r_{\text {spot }}<45^{\circ} .0$, where $r_{\text {spot }}$ is the angle subtended by the spot radius at the center of the white dwarf. To reproduce the light-curve amplitudes required temperatures of $\approx 11,000 \mathrm{~K}$ for the largest spots and $\approx 21,000 \mathrm{~K}$ for the smallest spots. Because of the influence the size of a spot can exert on the light-curve shape, we ran the same set of spot models for our three best-fitting models for the inclination and colatitude $\left(i=35^{\circ}, \beta=35^{\circ} ; i=45^{\circ}, \beta=35^{\circ}\right.$; and $i=40^{\circ}, \beta=40^{\circ}$ ). We find that the smallest $\chi_{\nu}^{2}$ values in all cases resulted when $i=35^{\circ}, \beta=35^{\circ}$, but the differences in the various $\chi_{\nu}^{2}$ values were very small. The best-fitting model has spot radius $r_{\text {spot }}=29^{\circ}$ ( $6 \%$ of the white dwarf's surface) and temperature $12,000 \mathrm{~K}$, but larger or smaller $\left(r_{\text {spot }} \pm 15^{\circ}\right)$ spots cannot be confidently excluded.

\subsection{Modeling the Infrared Light Curves with WD98}

Superficially, the $J$-band light curve of EF Eri resembles the optical light curves: it has a single large dip once per orbital period. However, the dips are deeper in the $J$ band than in the optical. The total amplitude for the best-fitting spot model in the optical was $\Delta m_{B}=0.19 \mathrm{mag}$, while the dips in the $J$ band are twice as deep. The $H$ - and $K$-band light curves also exhibit one maximum and one minimum per orbit, but now the minima are earlier by 0.4 in phase from their optical counterparts. The amplitude of the peakto-peak variations at $K$ is about $0.8 \mathrm{mag}$, while in the $H$ band the variations are $0.5 \mathrm{mag}$ in amplitude. Clearly, the same hot spot used to explain the optical data and possibly the $J$-band light curve cannot account for the $H$ - and $K$ band light curves.

In Figure 5 we attempted to deconvolve the SED of EF Eri to better derive the relative contributions of the secondary star in each of the infrared bandpasses to help constrain the light-curve modeling process. We used the blackbody fit to the white dwarf to remove its contribution from the observed fluxes. In Table 1, we have listed the observed fluxes at two different orbital phases for each bandpass and the estimated infrared fluxes of the white dwarf by using the $9500 \mathrm{~K}$ blackbody fit. We do this because the minima in $J$ band occur near orbital phase 0.5 , while the minima in $H$ and $K$ occur at phase 0.0. The last column in Table 1 gives the relative contribution of the white dwarf at the respective flux minimum observed in each of the infrared bandpasses. This table shows that the white dwarf is a minor component of the luminosity in $H$ and $K$, and any hot spot on its surface will not seriously affect the light curve at these two wavelengths. Interestingly, the white dwarf dominates the systemic luminosity during the $J$-band minima.

We first ran a variety of models using "normal" secondary stars $\left(T_{\text {eff }} \geq 2000 \mathrm{~K}\right)$ to attempt to generate the $H$ - and $K$-band light curves. These models incorporated the proper fractional contribution of the white dwarf in each bandpass. Such models resulted in light curves dominated by ellipsoidal variations (with peak-to-peak amplitudes of $\Delta J \approx \Delta H \approx \Delta K=0.12 \mathrm{mag}$ ). We then explored models with substantially cooler secondary stars. We found that as the temperature of the secondary dropped below $1600 \mathrm{~K}$, the ellipsoidal variations were gradually replaced by larger features that recurred once per orbital cycle. To explain the $H$ - and $K$-band light curves requires very cool secondary stars.

The explanation for this effect is that irradiation by the white dwarf heats one face of the secondary star. If one face is significantly hotter than the other, it will be more luminous, and when in view the system will be brighter. For example, if the amplitude of the $K$-band light curve is taken as $0.8 \mathrm{mag}$, the hotter hemisphere needs to produce twice

TABLE 1

Relative Contributions of the White DWarf in EF Eri

\begin{tabular}{|c|c|c|c|c|}
\hline Bandpass & $\begin{array}{l}\text { Observed Flux } \\
\text { Phase } 0.0 \\
\left(\mathrm{~W} \mathrm{~cm}^{-2}\right)\end{array}$ & $\begin{array}{l}\text { Observed Flux } \\
\text { Phase } 0.5 \\
\left(\mathrm{~W} \mathrm{~cm}^{-2}\right)\end{array}$ & $\begin{array}{c}\text { White Dwarf Flux } \\
\text { (No Spot) } \\
\left(\mathrm{W} \mathrm{cm}^{-2}\right)\end{array}$ & $\begin{array}{c}\text { Relative } \\
\text { Contribution } \\
\text { of WD at Min. }\end{array}$ \\
\hline ........... & $5.50 \times 10^{-20}$ & $3.88 \times 10^{-20}$ & $2.6 \times 10^{-20}$ & $67 \%$ \\
\hline$H$ & $8.34 \times 10^{-20}$ & $1.23 \times 10^{-19}$ & $1.3 \times 10^{-20}$ & $16 \%$ \\
\hline 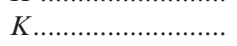 & $5.70 \times 10^{-20}$ & $1.09 \times 10^{-19}$ & $5.9 \times 10^{-21}$ & $10 \%$ \\
\hline
\end{tabular}


the luminosity of the cool hemisphere. The implied ratio of the temperature of the two sides is $T_{\text {hot }} / T_{\text {cool }}=1.20$. With the temperature, the luminosity, and the radial distance of the white dwarf being fairly well estimated, we can calculate whether there is sufficient energy impacting on the secondary star to drive such a temperature difference. We find that at the average distance of the secondary star's photosphere, the incident flux is $F_{\text {inc }}=3.47 \times 10^{8} \mathrm{ergs} \mathrm{s}^{-1} \mathrm{~cm}^{2}$. The temperature of the heated face of the secondary star $\left(T_{\text {hot }}\right)$ will readjust itself according to the relation $\sigma\left(T_{\text {hot }}\right)^{4}=$ $\sigma\left(T_{\text {cool }}\right)^{4}+(1-w) F_{\text {inc }}$ (where $w$ is the albedo and $T_{\text {cool }}$ is the nonirradiated temperature; see Brett \& Smith 1993). Assuming a typical convective star albedo of $w=0.5$ and using $T_{\text {hot }}=1.20 T_{\text {cool }}$ gives $T_{\text {cool }}=1300 \mathrm{~K}$, and therefore $T_{\text {hot }}=1600 \mathrm{~K}$. It is obvious that the actual temperatures could be lower than this depending on the chosen albedo and because the surface elements of the hemisphere that faces the white dwarf on the mildly distorted secondary star do not see the full flux because of the steep angle of incidence of the incoming photons. Obviously, however, there is sufficient flux being emitted by the white dwarf to drive a heating capable of explaining the observed light curve, and we have been able to successfully use this process to model both the $H$ - and $K$-band light curves of EF Eri.

\subsubsection{Irradiated Limb-darkening Coefficients and Albedos}

Before we attempted to run models for EF Eri that included the heating by the primary star, it was important to investigate the nature of the limb-darkening coefficients and the bolometric albedo in situations in which the secondary star is being irradiated. As stated earlier, even the nonirradiated versions of these quantities have yet to be tabulated for stars with temperatures below $2000 \mathrm{~K}$, and it will be necessary to extrapolate what is known to the temperature regime of interest. In light-curve modeling, two sets of limb-darkening coefficients are relevant. The first are the bolometric limb-darkening coefficients that help govern the heating/reflection effect (see Wilson 1990; Van Hamme 1993), and the second are the wavelength-specific limbdarkening coefficients that describe the emergent flux. In both cases we have chosen to use the square root law formalization for the limb-darkening coefficients since this parameterization seems to better model the emergent flux at infrared wavelengths (Van Hamme 1993).

Alencar \& Vaz (1999) have investigated how irradiation affects the limb-darkening coefficients for a number of scenarios involving a cool secondary star and a hot irradiating primary star. The effects of irradiation are dramatic, with limb brightening a common result of their calculations. The most similar model to the case of EF Eri is Alencar \& Vaz's model 136, in which the primary star is about $8000 \mathrm{~K}$ hotter than the secondary. We have extracted both the bolometric and wavelength-specific limb-darkening coefficients for model 136 and for those models with slightly hotter secondary stars to investigate how the limb-darkening coefficients change with the temperature of the secondary star. For secondary stars with temperatures less than $6000 \mathrm{~K}$, the coefficients change very slowly with temperature, thus providing some hope that they can be extrapolated below the range investigated by Alencar \& Vaz. The limb-darkening coefficients we have arrived at through extrapolation down to $2000 \mathrm{~K}$ are listed in Table 2 (where they are compared with the normal nonirradiated limb-darkening coefficients for
TABLE 2

Final Model Parameters

\begin{tabular}{|c|c|}
\hline Parameter & Value \\
\hline White dwarf temperature & $9500 \mathrm{~K}$ \\
\hline 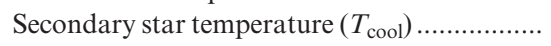 & $900 \mathrm{~K}$ \\
\hline Mass ratio $(q)$ & 0.10 \\
\hline Contribution of white dwarf at $J_{\min } \ldots \ldots \ldots \ldots \ldots$ & $67 \%$ \\
\hline Contribution of white dwarf at $H_{\min } \ldots \ldots \ldots \ldots \ldots . . . . . . .$. & $16 \%$ \\
\hline Contribution of white dwarf at $K_{\min } \ldots \ldots \ldots \ldots \ldots$ & $10 \%$ \\
\hline Contribution of secondary star at $J_{\min } \ldots \ldots \ldots \ldots$ & $<20 \%$ \\
\hline Contribution of secondary star at $H_{\min } \ldots \ldots \ldots .$. & $84 \%$ \\
\hline Contribution of secondary star at $K_{\min } \ldots \ldots \ldots \ldots$ & $90 \%$ \\
\hline $\begin{array}{l}\text { Extrapolated limb-darkening } \\
\text { coefficients at } \mathrm{H}\end{array}$ & \\
\hline Normal limb-darkening coefficients at $\mathrm{H}^{\mathrm{a}} \ldots \ldots$ & $x=0.080, y=0.924$ \\
\hline 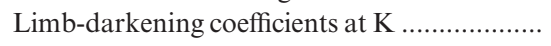 & $x=1.149, y=-1.660$ \\
\hline Normal limb-darkening coefficients at $\mathrm{K}^{\mathrm{a}} \ldots \ldots$ & $x=-0.131, y=1.037$ \\
\hline 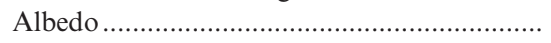 & 0.90 \\
\hline Hot spot longitude (orbital phase)...................... & 0.89 \\
\hline Hot spot latitude & $\beta=35^{\circ}$ \\
\hline Hot spot temperature & $12,000 \mathrm{~K}$ \\
\hline Hot spot radius .... & $29^{\circ}$ \\
\hline Orbital inclination & $i=45^{\circ}$ \\
\hline
\end{tabular}

${ }^{\text {a }}$ From Claret 1998, for $T_{\text {eff }}=2000 \mathrm{~K}$.

$T_{\text {eff }}=2000 \mathrm{~K}$ calculated by Claret 1998). We continued the extrapolation to lower temperatures, but the derived temperature dependence of the limb-darkening coefficients was relatively weak, and using these extrapolated values did not substantially change the final results. These extrapolated limb-darkening coefficients produced mild limb brightening.

In light-curve modeling programs, the albedo is usually set equal to 1 for radiative stars and to 0.5 for convective stars. As discussed in Nordlund \& Vaz (1990), the bolometric albedo can be affected by irradiation, and they find that values of $w=0.65$ are quite common. In these models, the irradiation heats the upper layers of the atmosphere and changes both the temperature structure and opacity. In modeling the light curves of three sdOB binary stars containing heavily irradiated low-mass low-luminosity secondary stars, Hilditch, Harries, \& Hill (1996) found that albedos of up to $w=1.0$ were necessary to explain their observations; thus, the irradiation of a cool star by a hotter one significantly changes the albedo, and in the following we will allow the albedo to assume values up to $w=1.0$. In addition Hilditch, Harries, \& Hill found that the model limb-darkening coefficients for the secondary stars in sdOB binaries were much smaller than normal or even produced limb brightening. Those authors believed that both effects likely arose because the irradiation has created an extended atmosphere above the heated face of the secondary star, and optical depth effects in this extended atmosphere differ dramatically from those of a normal stellar photosphere.

\subsubsection{Model Results in $H$ and $K$}

Using the extrapolated irradiated limb-darkening coefficients listed in Table 2 as inputs and allowing the albedo to assume large values, we investigated a wide range of models with WD98 in which the hemisphere of the secondary star that faces the white dwarf is substantially hotter than the nonirradiated face. As described above, given the incident flux $\left(F_{\text {inc }}\right)$ from the white dwarf we can predict the 
temperature of the hot side ( $\left.T_{\text {hot }}\right)$ of the secondary for any photospheric temperature $\left(T_{\text {cool }}\right)$. We ran models with a single heated hemisphere over the range $700 \mathrm{~K} \leq T_{\text {cool }} \leq 2000$ $\mathrm{K}$. In these models, we have incorporated our estimation for the contribution of the white dwarf to the observed fluxes in both bandpasses $(16 \%$ at $H$, and $10 \%$ at $K)$. We have assumed that the secondary star is responsible for the remaining infrared luminosity (i.e., $84 \%$ for $H$ and $90 \%$ for $K$ at phase 0.0 ). Models with relatively hot secondary stars, $T_{\text {cool }} \gtrsim 1800 \mathrm{~K}$, produced light curves that continue to be dominated by ellipsoidal variations. In such models, the heated face has $T_{\text {hot }} \gtrsim 2000 \mathrm{~K}$. As the temperature of the secondary star was reduced, the ellipsoidal light variations gave way to single-humped light curves in $H$ and $K$. The size and shape of these features were amplified by the use of the irradiated limb-darkening coefficients.

The fit of the model light curves to the $H$ - and $K$-band data improved as the temperature of the model secondary star became cooler. The first model (with $i=45^{\circ}$ ) that provided a good fit to the data was one with a secondary star temperature of $T_{\text {cool }}=900 \mathrm{~K}$ ! To get this model to fit, however, required an albedo of $w=0.90$. Cooler secondary star models fitted the data equally well and had lower albedos. For example, a $700 \mathrm{~K}$ model required an albedo of only 0.70 . We explored changing the orbital inclination to determine whether this would allow for significantly hotter secondary stars. We found that by using an orbital inclination of $i=60^{\circ}$, the best-fitting model had a secondary star with a temperature of $1000 \mathrm{~K}$. Above orbital inclinations of $60^{\circ}$ the width of the model's sinusoidal features broadened significantly and no longer provided good fits to the light curves. It might be possible to find a set of limb-darkening coefficients that could narrow the width of the light-curve humps, allowing these higher inclination models, but we did not fully explore that extensive parameter space. Our final best-fitting model (with $T_{\text {cool }}=900 \mathrm{~K}$ ) has been plotted in Figure 2. Note that the limb-darkening coefficients used to generate this model are from Alencar \& Vaz (1999), extrapolated to $2000 \mathrm{~K}$. We attempted more extreme values for the limb-darkening coefficients, calculated by continuing the extrapolation down to $900 \mathrm{~K}$, but the changes in the shape and the amplitude of the light curves were not substantially different from those derived using the values extrapolated to $2000 \mathrm{~K}$. The extremely limited range in temperature over which we could find suitable models is surprising. We believe the main reason for this is that to drive the large amplitude variations seen in the $K$ band the spectral energy distribution of the hot side of the secondary star has to peak in the $K$ band, and this requires a temperature of $T_{\text {eff }} \approx 1600 \mathrm{~K}$.

\subsubsection{Modeling the J-Band Light Curve}

With its resemblance to the optical light curves, it seems logical to produce the $J$-band light curve in the same fashion. But as previously noted, the $J$-band light curve has deeper minima. The amplitude of the variations in the $J$ band from the photospheric hot spot cannot be larger than those seen in the optical. To reproduce the depth of the $J$-band minima therefore requires an additional source of luminosity at roughly the same phase as the hot spot on the white dwarf's surface. It also must supply a similar amount of additional luminosity. We believe the source of this luminosity is the cyclotron emission associated with the magnetic pole region. As shown in Ferrario et al. (1996), the strongest of the cyclotron features is located in the $J$ band. Residual cyclotron emission during quiescence needs to produce only an additional $\approx 25 \%$ of the white dwarf's luminosity to explain the $J$-band minimum (this type of contribution during a low state has been seen in other polars; see Howell et al. 2000). We artificially elevated the luminosity of the white dwarf hot spot used to explain the optical light curves so as to mimic this cyclotron component and produced the model $J$-band light curve plotted in Figure 1 . Note that if there is residual cyclotron emission in the $J$ band, the $H$ and $K$ bands should also contain some cyclotron flux. The high-state infrared spectrum shows that the continuum in the $H$ band is $80 \%$ of that in the $J$ band, dropping to $50 \%$ of the $J$ band at $K$. If this ratio is maintained at minimum, then the cyclotron emission in the $H$ band would be about $3 \%$ of the observed flux (at phase 0.0 ), even less at $K$. This can be more easily envisioned by examining the high-state SED plotted in Figure 5 and sliding it down until the high-state $J$-band flux matches the quiescent (white dwarf-subtracted) $J$-band flux. If the shape of the high-state cyclotron-dominated SED is maintained into quiescence, the $H$ - and $K$-band light curves should have very low levels of contamination from this process.

\section{RESULTS}

We have been able to successfully model the BVRIJHK light curves of EF Eri through the use of a hot spot on the white dwarf surface and a very cool irradiated brown dwarf-like secondary star. The model, evolved to explain the optical light curves, appears robust. It is logical to assume that the site of the impact of the accretion column on the white dwarf surface is hotter than the surrounding regions if mass accretion is still occurring. Even if the mass transfer has ceased, the cooling rates for the white dwarfs and their accretion regions can be quite slow. For example, Gänsicke, Beuermann, \& Martino (1995) could not find evidence for cooling of the hot spot accretion region in the prototype polar AM Her during its prolonged low states. Continued optical monitoring would provide a sensitive test for any cooling of the accretion hot spot. Especially useful in this regard would be phase-resolved $U$-band data to help better differentiate the hot spot flux from that of the underlying photosphere.

The infrared light curves were more difficult to model. The $J$-band light curve resembled the optical light curves, except the minima were substantially deeper. To create these deeper $J$-band minima required the hot spot to have twice the luminosity of the optical hot spot. As shown by Ferrario et al. (1996), when EF Eri is in its high state, the strongest cyclotron feature is in the $J$ band; thus it appears that there is some residual cyclotron emission during the current low state and hence, possible low-level mass accretion. In our best-fitting models to the $J$-band light curves the minima were slightly shifted in phase from the optical minima. The minima of the $J$-band models occurred at an orbital phase near 0.5 , but the best-fitting models to the optical data had minima centered at an orbital phase of 0.4 . Given the considerable structure in the light curve and the modest $\mathrm{S} / \mathrm{N}$ of the $J$-band photometry, it is difficult to determine the phase of these minima with high precision. But it is clear that the minima in the $J$ band occur well after those seen in the optical data. One interpretation is that the residual cyclotron 
emission is coming from a region located above the accretion hot spot (see Howell et al. 2001a or Schwope et al. 2001).

The most remarkable aspect of the multiwavelength light curves of EF Eri is the transition as one proceeds from the $J$ band to the $H$ band: where there are minima in the $J$ band, there are maxima at $H$ and $K$ ! We were able to reproduce the $H$ - and $K$-band light curves, but to do so required a very cool irradiated secondary star. The amplitude of the variations in the $K$ band are larger than in the $H$ band, suggesting that the spectral energy distribution of the irradiated secondary must peak redward of $H$. This implies that the effective temperature of the irradiated hemisphere of this object has $T_{\text {eff }} \lesssim 1700 \mathrm{~K}$. If so, the spectrum of such an object should appear similar to those of early L-type dwarfs. Our $K$-band spectrum of EF Eri is consistent with this result in that it clearly shows a prominent decline on the red end of the spectrum due to the expected absorption from the combination of $\mathrm{CO}$ and $\mathrm{H}_{2} \mathrm{O}$ features. As demonstrated in Figure 6, this orbitally averaged $K$-band spectrum is dominated by flux from the irradiated hemisphere of the secondary star and thus is not that of the underlying object. Our model suggests that the spectrum of the cooler hemisphere of the secondary star should resemble a late-L/early $\mathrm{T}$ dwarf, but we were unable to extract a useful spectrum during the $K$-band minima to test this proposition. The deconvolved spectral energy distribution (Fig. 5), however, is consistent with a very late-type L dwarf.

The blueward rise seen in the $K$-band spectrum of EF Eri was unexpected, given the fact that late-type stars have a strong water vapor absorption feature centered near 1.90 $\mu \mathrm{m}$ (as is demonstrated in the spectrum of VB 10, where the flux declines blueward of $2.15 \mu \mathrm{m}$ ). However, as we derived

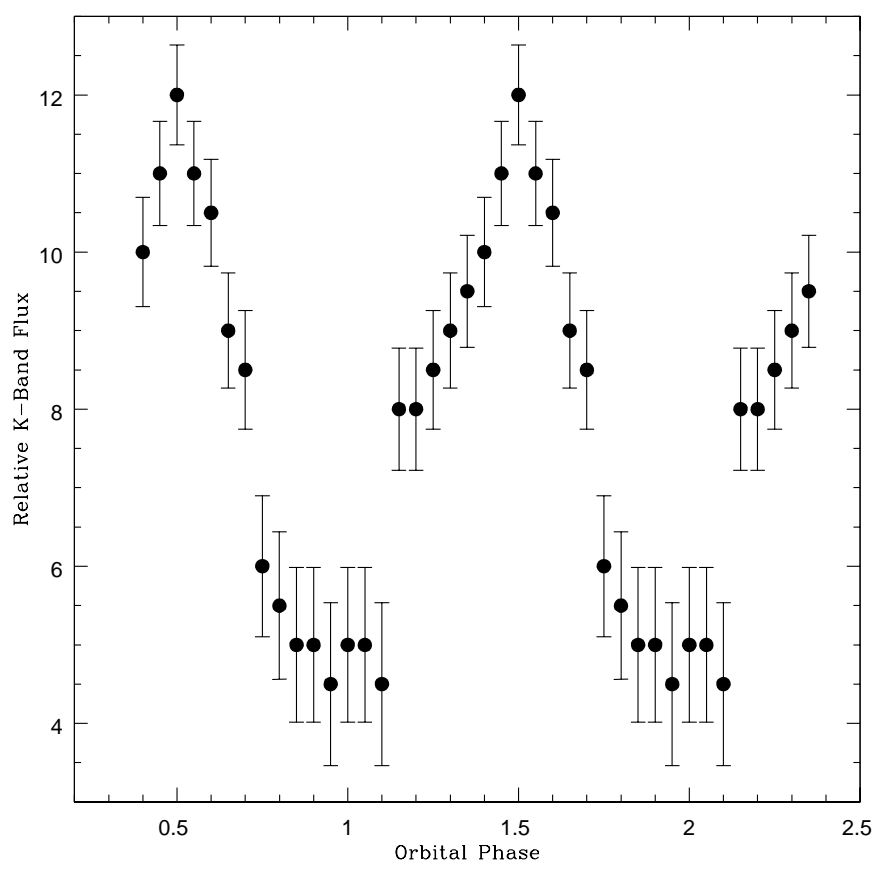

FIG. 6.- Spectrum shown in Fig. 3 obtained two months before the infrared photometry shown in Fig. 1, resulting from the combination of a large number of individual spectra obtained over several hours. These individual spectra can be used to generate a $K$-band light curve by summing the flux in each of these spectra. The result (folded for clarity) reveals the same largeamplitude variations as seen in the $K$-band photometry. earlier (see Table 1), depending on orbital phase the white dwarf in EF Eri contributes between $5 \%$ and $10 \%$ of the $K$ band flux; thus, we can estimate the contribution of the white dwarf to the spectrum, assuming it supplies this fraction of the $K$-band flux. In Figure 3 we have plotted the spectrum of a $9500 \mathrm{~K}$ blackbody normalized so that it has $7.5 \%$ of EF Eri's $K$-band flux. It is extremely difficult to choose the level of the white dwarf contribution in this comparison given the fact that the $K$-band spectrum was generated through the co-addition of spectra obtained over two orbital cycles. Even in this rough comparison, however, it is apparent that the blueward rise in the spectrum can be easily attributed to the white dwarf.

\subsection{Relation between Bailey Phase and Binary Orbital Phase}

The ephemeris derived for EF Eri by Bailey et al. (1982) has the zero arbitrarily defined by a narrow dip feature seen in the high-state $J$ and $K$ bands with an apparently related but much broader dip seen in the optical. Given that the accretion stream leads the secondary star, any accretion region eclipse within the gas stream (leading to narrow dips in the light curve) should occur prior to inferior conjunction (phase 0.0) of the secondary star. A self-eclipse of the accretion region by the white dwarf should therefore be centered $180^{\circ}$ later. Bailey et al. center the self-eclipse of the accretion region by the white dwarf at phase 0.4 , and by analogy our most direct view of the accretion region, where the narrow dips should be centered, would then occur near phase 0.9. Bailey et al. note this discrepancy and ascribe it to an asymmetry in the field geometry. Our light-curve modeling, however, appears to force us to reconcile the fact that we see the center of the heated face of the secondary star at phase 0.5 of Bailey et al. and thus true orbital phase 0.0 is the same (or nearly so) as phase 0.0 of Bailey et al. Additionally, it appears that the phase of self-eclipse $(0.4)$ is constant in both the phasing of Bailey et al. and our light-curve modeling and is not diametrically opposite from phase 0.0; thus, the standard assumption for polars about the relationship between the narrow dip, the self-eclipse feature, and the true orbital phase does not appear to work for EF Eri.

To attempt a reconciliation of the orbital phase of our light-curve models with the phasing of EF Eri determined by Bailey et al. (1982), we first make a few general assumptions about EF Eri. Observations of polars often show a narrow dip feature such as that seen and used by Bailey et al. to phase the light curves of EF Eri. These narrow dips are believed to be due to an eclipse of the accretion pole region by the far-field accretion stream (see Sirk \& Howell 1998, and references therein). This eclipse is produced by the incoming stream after it becomes magnetically controlled and is lifted out of the orbital plane. Assuming that EF Eri's white dwarf is still tidally locked to the orbit even during this extended low state, ${ }^{5}$ we can model the accretion stream within the system and determine where, in true orbital phase, the narrow dip used as phase 0 by Bailey et al. will likely occur.

Figure 7 presents a three-dimensional smooth-particle hydrodynamics model for EF Eri, based on the work of Cash (2002). The model was run with a mass transfer rate of

\footnotetext{
${ }^{5}$ Note that our light-curve analysis in $\S 4$ shows that the white dwarf has remained locked.
} 

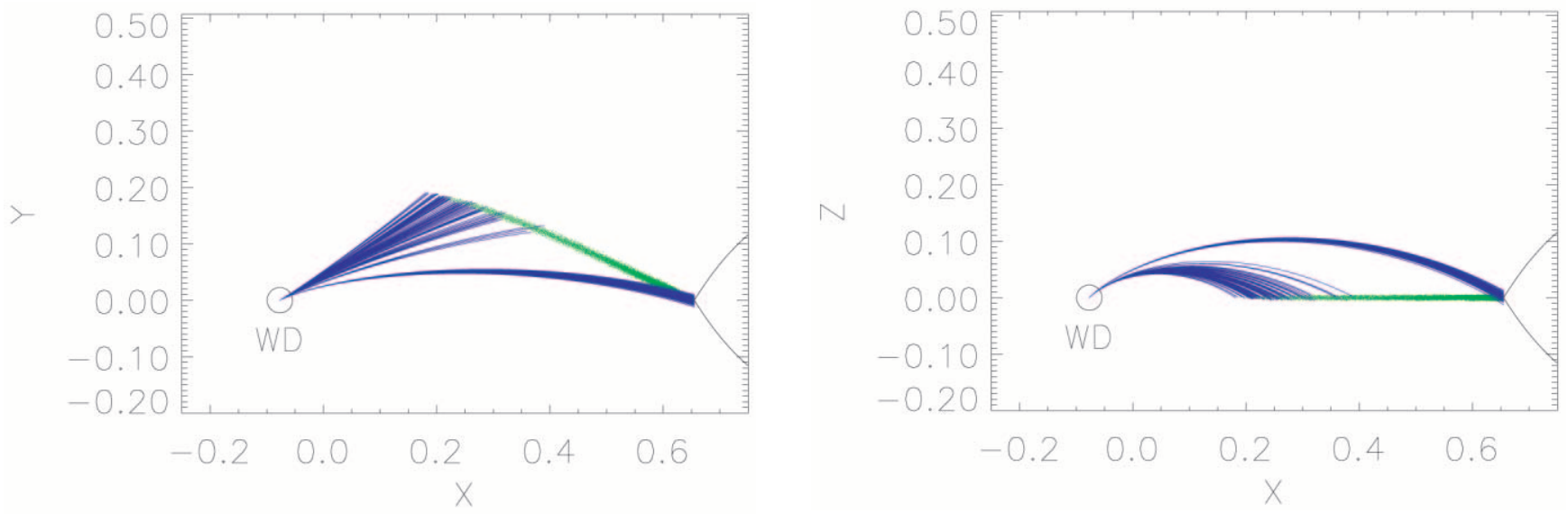

FIG. 7.- Smooth-particle hydrodynamic model for the high-state accretion stream of EF Eri, based on the known system parameters used to model the photometry of EF Eri, showing the ballistically controlled accretion stream (green) and the magnetically controlled stream (blue). Left, $X$ - $Y$ or orbital plane view; right, view in the $X-Z$ plane. The white dwarf is drawn to scale, and a part of the Roche lobe of the secondary (near $L_{1}$ ) is shown at right. We find that for EF Eri during a high state there is essentially no ballistic stream and that $60 \%$ to $80 \%$ of the material is captured directly at $L_{1}$.

$3.2 \times 10^{-11} M_{\odot} \mathrm{yr}^{-1}$ (predicted to be the high-state transfer rate; see Howell et al. 2001b) and the other system parameters used herein. The model shows the location of the gas stream within the binary in both $X-Y$ (orbital) and $X-Z$ planes. The accretion material is coded as to when it is ballistically controlled (green), or when it is magnetically controlled (blue). For the very close separation of the white dwarf and low-mass secondary in EF Eri, our models indicate that the magnetic field captures more than half the accreted material directly at $L_{1}$. This means that during its high state the accreted material in EF Eri will be a geometrically narrow dense stream flowing directly from $L_{1}$ to the magnetic pole region on the white dwarf surface. The shallow angle of the accretion stream to our line of sight allows an observer to see an eclipse of the accretion region by the stream very close to true orbital phase 0 (predicted in these models to occur near binary phase 0.98 ).

The EF Eri system is therefore unusual in that there is essentially no ballistic stream and that phase 0.0 defined by the narrow stream eclipse is almost exactly equal to true binary orbital phase. These models allow us to reconcile the difficulties with the phasing discussed above and show how the pole and antipole directions can appear to be separated by an angle not equal to $180^{\circ}$ : The gas stream never makes it very far "downstream" to where it would then be magnetically controlled, bent out of the orbital plane, and then forced back toward the white dwarf (as is seen in most polars); thus, the narrow stream eclipse does not occur near the usual orbital phase 0.9 but very near phase 0.0 . It is interesting to note that Allen \& Cherepashchuk (1982) found that the accretion column of VV Pup was advanced by only 0.05 in phase from that of inferior conjunction.

\subsection{Future Work}

EF Eri is an ideal target to examine the nature of the secondary star in one of the shortest-period CV systems. Our $K$-band spectrum of EF Eri clearly indicates the presence of a very late-type secondary star. This spectrum, however, represents only the hotter hemisphere of the secondary star. Both the results of the light-curve modeling and spectral energy distribution suggest that the secondary star is L8 or later. Mennickent \& Diaz (2002) have combined the results of recent evolutionary code predictions from Howell et al. (2001b) with new and existing limits on the spectral types of the secondary stars for some of the shortest-period CVs to produce an updated $T_{\text {eff }}$ versus orbital period diagram (see their Fig. 7). From this diagram, the predicted temperature for the secondary star in EF Eri is $T_{\text {eff }} \approx 1000 \mathrm{~K}$, completely consistent with our results. $K$-band spectroscopy during minimum will be required to confirm this, but will require an $8 \mathrm{~m}$ class telescope. In addition, new higher $\mathrm{S} / \mathrm{N}$ optical photometry can provide critical insight into the rate of cooling of the hot spot. Given the dramatic fading of the hydrogen emission lines, it should now also be possible to directly measure the radial velocity curve of the white dwarf primary using high-resolution optical spectroscopy. These types of data would provide valuable insight into the nature of both components in this short-period system.

Finally, it is relevant to note how difficult it would be to identify objects such as EF Eri in surveys to find the "missing" population of short-period CVs. The optical colors of EF Eri are not very blue, for example, $B-V \approx V-I \approx 0.1$. There is also almost no trace of the secondary star shortward of the $H$ band, nor are there any significant signatures of mass accretion. It is possible that the majority of the postperiod minimum CVs evolve to states that resemble EF Eri, making them very difficult to identify in optical surveys. If one wishes to identify quiescent systems similar to EF Eri, then one needs to find objects that have the colors of $A$ dwarfs in the optical but have the $H-K$ colors of $L d$ warfs!

We would like to acknowledge Peter Hauschildt for useful discussions regarding late-type stars and their atmospheres. We would like to thank Boris Gänsicke for providing us with his white dwarf model. T. E. H. acknowledges partial support under NSF grant AST 99-86823, H. L. O. has received support under LANL subcontract 29676-00101 37, and S. B. H. acknowledges partial support of this research under NSF grant AST 98-10770. The United Kingdom Infrared Telescope is operated by the Joint Astronomy Centre on behalf of the UK Particle Physics and Astronomy Research Council. 
Alencar, S. H. P. \& Vaz, L. P. R. 1999, A\&AS, 135, 555

Allen, D. A., \& Cherepashchuk, A. M. 1982, MNRAS, 201, 521

Bailey, J., Hough, J. H., Axon, D. J., Gatley, I., Lee, T. J., Szkody, P.,

Stokes, G., \& Berriman, G. 1982, MNRAS, 199, 801

Bessell, M. S., \& Brett, J. M. 1988, PASP, 100, 1134

Beuermann, K., Wheatley, P., Ramsay, G., Euchner, F., \& Gänsicke, B. T. 2000, A\&A, 354, L49

Brett, J. M., \& Smith, R. C. 1993, MNRAS, 264, 641

Carpenter, J. M. 2001, AJ, 121, 2851

Cash, J. L. 2002, Ph.D. thesis, Univ. Wyoming

Claret, A. 1998, A\&A, 335, 647

Cropper, M. 1985, MNRAS, 212, 709

1990, Space Sci. Rev., 54, 195

Ferrario, L., Bailey, J., \& Wickramasinghe, D. 1996, MNRAS, 282, 218

Gänsicke, B. T., Beuermann, K., \& de Martino, D. 1995, A\&A, 303, 127

Gelino, D. M., Harrison, T. E., \& McNamara, B. J. 2001a, AJ, 122, 971

Gelino, D. M., Harrison, T. E., \& Orosz, J. A. 2001 b, AJ, 122, 2668

Harrison, T. E., Howell, S. B., Osborne, H. O., Johnson, J. J., \& Gelino,

D. M. 2003, in preparation

Harrison, T. E., Johnson, J. J., \& Howell, S. B. 2002, in ASP Conf. Ser. 262,

The Physics of Cataclysmic Variables and Related Objects, ed.

B. Gansicke, K. Beuermann, \& K. Reinsch (San Francisco: ASP), 57

Hilditch, R. W., Harries, T. J., \& Hill, G. 1996, MNRAS, 279, 1380

\section{REFERENCES}

Howell, S. B., \& Ciardi, D. R. 2001, ApJ, 550, L57

Howell, S. B., Ciardi, D., Dhillon, V., \& Skidmore, W. 2000, ApJ, 530, 904

Howell, S. B., Gänsicke, B., Szkody, P., \& Sion, E. 2002, ApJ, 575, 419

Howell, S. B., Gelino, D. M., \& Harrison, T. E. 2001a, AJ, 121, 482

Howell, S. B., Nelson, L. A., \& Rappaport, S. 2001b, ApJ, 550, 897

King, A. R., \& Cannizzo, J. K. 1998, ApJ, 499, 348

McLean, I. S., Prato, L., Kim, S. S., Wilco, M. K., Kirkpatrick, J. D., \& Burgasser, A. 2001, ApJ, 561, L115

Mennickent, R. E., \& Diaz, M. P. 2002, MNRAS, 336, 767

Nordlund, A., \& Vaz, L. P. R. 1990, A\&A, 228, 231

Östreicher, R. Seifert, W., Wunner, G., \& Ruder, H. 1990, ApJ, 350, 324

Schwope, A. D., Schwarz, R., Sirk, M., \& Howell, S. B. 2001, A\&A, 375, 419

Sirk, M., \& Howell, S. B. 1998, ApJ, 506, 824

Van Hamme, W. 1993, AJ, 106, 2096

Wheatley, P. J., \& Ramsay, G. 1998, in ASP Conf. Ser. 137, Wild Stars in the Old West, ed. S. Howell, E. Kuulkers, \& C. Woodward (San Francisco: ASP), 446

Wilson, R. E. 1990, ApJ, 356, 613

2. 1998, Reference Manual to the Wilson-Devinney Program, Computing Binary Star Observables, Version 1998 (Gainsville: Univ. Florida) 